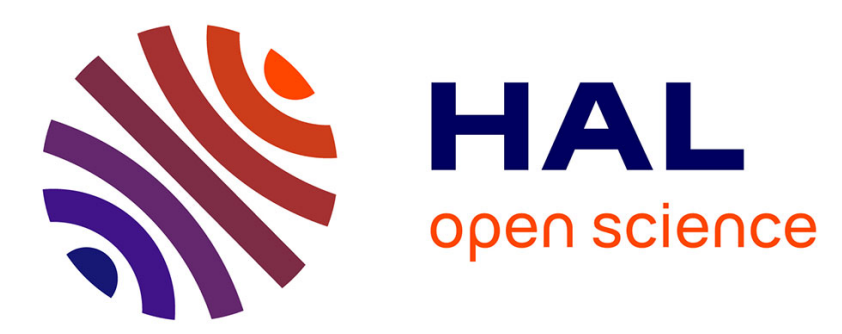

\title{
Instruire les risques professionnels en entreprise : étude d'un travail collaboratif d'intégration des risques
}

Ludovic Tendille, Valérie Saint-Dizier de Almeida, Corinne van de Weerdt

\section{To cite this version:}

Ludovic Tendille, Valérie Saint-Dizier de Almeida, Corinne van de Weerdt. Instruire les risques professionnels en entreprise : étude d'un travail collaboratif d'intégration des risques. Psychologie du travail et des organisations, 2017, 23 (4), pp.380-393. 10.1016/j.pto.2017.08.001 . hal-02042362

\section{HAL Id: hal-02042362 \\ https://hal.science/hal-02042362}

Submitted on 18 Oct 2019

HAL is a multi-disciplinary open access archive for the deposit and dissemination of scientific research documents, whether they are published or not. The documents may come from teaching and research institutions in France or abroad, or from public or private research centers.
L'archive ouverte pluridisciplinaire HAL, est destinée au dépôt et à la diffusion de documents scientifiques de niveau recherche, publiés ou non, émanant des établissements d'enseignement et de recherche français ou étrangers, des laboratoires publics ou privés. 


\title{
Instruire les risques professionnels en entreprise : étude d'un travail collaboratif d'intégration des risques Teach professional risks in companies: Study of a collaborative work of psychosocial risks' integration.
}

\author{
Ludovic Tendille*, Valérie Saint-Dizier de Almeida** \& Corinne Van de Weerdt*** \\ * Psychologue du travail, Centre d'Information et d'Orientation, 13 rue du $10^{\text {ème }}$ BCP, 88100 Saint-Dié-des-Vosges, France, \\ tél : 0627208613, mail : ludovic.tendille@ac-nancy-metz.fr \\ ** Professeur, Université de Lorraine, 23 bd Albert 1 ${ }^{\text {er }}$ BP 13397, 54015 Nancy Cedex, France, \\ mail : valerie.saint-dizier@univ-lorraine.fr \\ *** Responsable d'études, INRS, Laboratoire Ergonomie et Psychologie Appliquées à la Prévention, Rue du Morvan, CS \\ 60027, 54519 Vandœuvre Lès Nancy Cedex, mail : corinne.vandeweerdt@inrs.fr
}


L'évaluation des risques professionnels doit prendre en compte les risques psychosociaux au même titre que les risques physiques. L'article vise à montrer que la phase d'identification des risques physiques, lorsqu'elle implique des collectifs d'opérateurs concernés, est propice à la mise en exergue de risques psycho-sociaux. Nous montrons comment la régulation des risques physiques nécessite la régulation des risques psychosociaux. Pour l'étayer, nous analysons une séquence extraite de la transcription d'une réunion dans une entreprise de productions de mailings publicitaires. L'étude débouche sur des préconisations relatives à la formation des instructeurs quant au traitement de l'ensemble des risques professionnels.

\section{Abstract}

The evaluation of professional risks has to take the psychosocial hazards into account as well as the physical hazards. The article aims at showing that the identification phase of physical hazards, when it implies relevant operator collectives, is favourable to the highlighting of situations with psychosocial risks. We show how the regulation of physical hazards needs the regulation of psychosocial hazards. To underpin it, we analyze sequences extracted from the transcription of a reunion in a company of advertising mailings' production. The study results in preconisation related to the instructors' program regarding the treatment as a whole of professional hazards.

Mots-clés : risques psychosociaux, risques physiques, animation, collectif, analyse de discours.

Keywords : psychosocial risk, physical risk, animation, collective, speech analysis. 


\section{Introduction}

La prévention des risques dans le monde du travail consiste à mettre en place des actions pour éviter ou limiter l'apparition des risques professionnels, et s'ils sont présents, pour les réduire et si possible les supprimer. Elle recouvre l'ensemble des dispositions à appliquer pour préserver la santé et la sécurité des salariés, améliorer les conditions de travail et tendre au bien-être au travail. En démarche de prévention, l'évaluation des risques revient à apprécier l'exposition au danger afin de prioriser les actions de prévention à mener. Les résultats de l'évaluation des risques doivent être formalisés (R.4121-1 du Code du travail) pour pouvoir être transcrits dans un Document Unique d'Evaluation des Risques (DUER) (Andéol, Guillemy \& Leroy, 2010).

La problématique s'inscrit dans le champ de l'évaluation des risques professionnels. En référence à la littérature, le processus d'évaluation procède généralement en deux étapes :

- La première vise l'identification de risques. Pour l'identification des risques psychosociaux, on recourt à des questionnaires comme SATIN (Kop, Grosjean, Formet \& Althaus, 2013), celui de Karasek et Théorell (1990). Pour les risques physiques, on peut utiliser par exemple la grille de positionnement en santé et sécurité au travail (grille GPS\&ST 2011) (Rossignol, Drais \& Favaro, 2016) ou l'outil de diagnostic en prévention pour la petite entreprise (grille DIGEST) (Malenfer, 2014). Ainsi on dispose d'outils spécifiques aux risques psychosociaux versus risques physiques. Les outils pour l'identification des risques psychosociaux se basent sur des théories excluant généralement les déterminants physiques et ceux utilisés pour l'identification des risques physiques tendent à minorer les déterminants psychologiques.

- La seconde étape vise à prioriser les risques identifiés et à trouver des solutions visant à les réduire ou à les supprimer. Dans ce cadre, on emploie de plus en plus de méthodes dites qualitatives (Jeoffrion, Hamard, Barre \& Boudoukha, 2014) reposant bien souvent sur la mise en place de réunions de groupe (Althaus, Grosjean \& Brangier, 2012). Des études ont en effet montré les effets limités des méthodes quantitatives centrées sur la phase de diagnostic au profit de méthodologies qualitatives davantage inscrites dans un continuum d'efficacité des actions de changement (Buono \& Jamieson, 2010 ; Althaus, Grosjean \& Brangier, 2012). Ces deux approches s'avèrent néanmoins complémentaires (Jeoffrion, Hamard, Barre \& Boudoukha, 2014). Les données qualitatives permettraient de prioriser la réflexion ou les actions à mettre en œuvre (Dumez, 2011).

On note ainsi qu'au moins pour la première étape, le traitement des risques psychosociaux et des risques physiques est différent ; sans doute d'une part parce que la législation n'a introduit que tardivement l'intégration des risques psychosociaux au DUER et d'autre part parce que leur identification a donné lieu à des outils différenciés.

L'enjeu de cette étude est de montrer que cette dichotomie ne devrait pas transparaître dans la phase de compréhension des situations à risque et leur régulation, car des troubles psychosociaux, des défauts organisationnels, des ambiances délétères... peuvent augmenter le risque d'incident ou d'accident (COCT, 2013 ; DARES, 2014) ; elle montre aussi que des risques pour la santé physique des opérateurs peuvent être causés par des composants socio-émotionnels et concourir à l'apparition de troubles psychosociaux (stress, crainte, appréhension...). La prévention des risques professionnels est optimisée par un management intégré de tous les risques de manière interactive.

Dans l'étude de cas qui nous occupe, les deux phases (identification et régulation) sont fusionnées. Il s'agit d'une réunion qui vise, sur la base du DUER à actualiser, à traiter des risques identifiés antérieurement, à en soumettre de nouveaux, à traiter de leur importance et proposer des solutions pouvant les réduire ou les supprimer. Si le DUER en question comporte uniquement des risques physiques, nous voulons montrer à l'instar d'autres auteurs que ce type de réunion est propice à l'investigation à la fois des risques physiques et des risques psychosociaux (Sarnin, Caroly \& Douillet, 2011 ; Aublet-Cuvelier, 2011 ; Toulouse \& al., 2013 ; Stock \& al., 2013) ; et que l'animateur doit être attentif à l'expression de risques psychosociaux, car comme nous allons le voir, ils peuvent aussi impacter la régulation des risques physiques. 
Le cas traité est celui d'une réunion d'évaluation des risques professionnels au sein d'une entreprise de production de mailings publicitaires visant l'actualisation du DUER. L'enjeu initial était d'identifier la structure fonctionnelle de ce type de réunion. Ce sont les résultats de l'analyse d'une séquence de ce corpus qui a conduit à la problématique traitée dans cet article. En l'occurrence l'analyse révèle que la régulation d'un risque physique peut impacter le climat social de la situation de travail.

Nous débutons par présenter le contexte de la réunion puis son analyse structuro-fonctionnelle afin d'identifier l'inscription de la séquence étudiée. Cette dernière fait ensuite l'objet d'une analyse interlocutoire grâce à laquelle les déterminants de la situation de danger sont identifiés ; ces déterminants sont formalisés grâce à la méthode de l'arbre des causes (Monteau, 1974).

\section{Le contexte : une réunion d'évaluation des risques professionnels}

\subsection{Une entreprise de production de mailings publicitaires}

La réunion d'évaluation des risques professionnels étudiée a eu lieu dans une entreprise de production de mailings publicitaires. Les activités principales de l'atelier - qui constitue l'unité de production - sont la mise sous pli et le tri postal. La mise sous pli est exécutée par les conducteurs qui doivent contrôler et alimenter en documents papiers des machines qui produisent des plis sortants, c'est-à-dire des enveloppes fermées comprenant l'ensemble des documents. Le tri postal est accompli par les routeurs qui reçoivent ces plis et les trient en fonction des codes postaux. Les conducteurs et les routeurs travaillent continuellement en position debout et peuvent être amenés à marcher le long de la machine. Les autres acteurs de l'atelier sont les chefs d'équipe qui supervisent la production et les approvisionneurs chargés notamment d'apporter les documents nécessaires à la production. Ces documents sont entreposés sur des palettes, à disposition des conducteurs. La mise en œuvre de la production au sein de l'atelier est permise grâce à l'articulation de trois autres unités de travail : le magasin, la maintenance et l'administration. Le magasin est l'unité de stockage des documents et des plis sortants de l'atelier. L'unité de maintenance comprend la maintenance préventive (suivre les préconisations des constructeurs) et la maintenance corrective (réparer lorsqu'une panne se présente). Enfin, l'administration constitue l'unité de gestion de l'entreprise.

\subsection{Le groupe de travail : différents acteurs impliqués dans l'actualisation du DUER}

Chaque année, conformément aux obligations légales, l'entreprise procède à l'évaluation des risques professionnels pour l'ensemble des unités de travail afin de mettre à jour le DUER. Pour mener cette opération, différents participants sont convoqués par le Responsable Production, Sécurité et Maintenance pour une réunion de travail par unité. L'animateur constitue un groupe de travail en sélectionnant de façon aléatoire les opérateurs qui n'ont pas participé à la réunion l'année précédente. Le collectif étudié ici est composé de quatre membres : trois opérateurs qui occupent les postes de conducteurs et de routeurs, et un animateur qui est le Responsable Production, Sécurité et Maintenance. Ce responsable dirige la production, la planification, et assure le management des chefs d'équipe. Il gère la maintenance de l'entreprise qu'elle soit préventive ou corrective, et s'occupe de la sécurité, du respect des affichages obligatoires, de l'entretien de divers éléments (bâtiment, électricité, extincteurs, etc.) y compris l'entretien des composants des machines de production. Il effectue aussi des exercices d'évacuation, de la formation, des plans d'intervention et réalise le processus d'évaluation des risques. Notons qu'il occupait auparavant le poste de conducteur dans l'atelier.

L'étude s'intéresse alors à un groupe restreint puisqu'il comporte peu de membres ayant des rôles différenciés pour l'atteinte d'un but commun (Anzieu \& Martin, 1968). Ce groupe restreint est momentané (Mucchielli, 1992) puisqu'il s'agit d'une réunion imposée et créée le temps de réaliser l'activité d'évaluation, événement inhabituel pour les membres du groupe.

\subsection{Le DUER de l'atelier est exploité par le collectif}

Le collectif pour cette réunion dispose d'un extrait du DUER de l'année précédente se rapportant à l'atelier. L'objectif affiché est de coter les risques présents. Pour déterminer la valeur d'un risque et de son impact associé, quatre critères sont retenus : 1) la fréquence d'apparition du risque lors du fonctionnement normal de 
l'activité de l'unité de travail - la note sera la plus élevée si l'opérateur est soumis au risque de manière permanente ;2) la probabilité d'apparition d'un dommage qui est liée au nombre d'accidents lors des deux dernières années ;

3) la gravité des conséquences pour la santé des salariés de l'accident ou de la maladie causée ; 4) le niveau de maîtrise du risque par l'organisation qui renvoie aux moyens mis en œuvre pour limiter ou annuler l'impact associé. A l'aide de ces critères, l'animateur obtient une note finale pour chaque risque. Les risques sont alors classés sur cette base pour chaque unité de travail. Ces classifications sont utilisées pour prioriser les actions de prévention ou de régulation des risques.

Le DUER comprend différentes catégories de risque (par exemple « risque de chute de plain-pied»). Pour chacune d'elles, des questions ciblent une situation précise (par exemple « y a-t-il possibilité de heurt ou de chute pour accéder au poste de travail ?») et différents dangers - identifiés l'année précédente - sont énumérés pour chacune des questions (par exemple «passe-câbles au sol, palettes trop rapprochées »). Pour chaque situation ciblée, introduite par une question, différents scores pointent les quatre critères évoqués précédemment et la note finale.

\section{Méthodologie}

\subsection{Le recueil de données}

La réunion a été enregistrée à l'aide d'un dictaphone avec l'accord écrit et oral des personnes au préalable. Les productions verbales sont transcrites dans leur littéralité et au plus près de leur prononciation (par exemple «

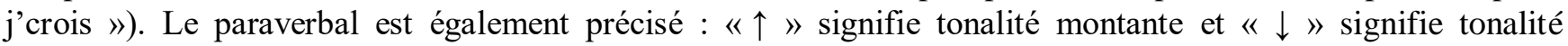
descendante. Les actions non verbales sont précisées entre parenthèses, par exemple «(A écrit sur le document) », « $(\mathrm{A}$ regarde $\mathrm{B}) »$ ou encore « (rire) ». Les pauses inférieures à une seconde sont marquées par le symbole « (.) »; la durée des pauses plus longues est indiquée en secondes (une pause de deux secondes est transcrite « (2') »).

\subsection{Méthodologies d'analyse}

L'étude du corpus repose sur une approche dialogique et pragmatique du discours qui suppose pour l'analyste d'être baigné dans l'espace interactionnel (Lacoste, 1983) afin de comprendre - autant que faire se peut - ce qui se produit et éviter dans une certaine mesure des erreurs d'interprétation. Cela suppose notamment de s'enquérir du cadre institutionnel, du statut des partenaires dans l'organisation et dans le cadre de cette réunion, de la consigne qui leur a été communiquée, de la tâche qu'ils ont à accomplir collectivement.

L'étude de la dimension structuro-fonctionnelle de l'activité du groupe repose sur une conception gestaltiste de la communication selon laquelle toute communication possède une organisation, une architecture qui se façonne et se révèle au gré des productions langagières (Kostulski \& Trognon, 1998); elle exploite les outils développés par les linguistes de l'Ecole de Genève (Roulet, Auchlin, Moeschler, Rubbattel, \& Schelling, 1985). L'analyse structuro-fonctionnelle permet d'identifier les différentes phases de l'activité sur la base de leur fonctionnalité et leur articulation. Cette forme d'analyse permet au chercheur d'avoir une vision de l'activité globale dans sa dimension structuro-fonctionnelle et d'engager des analyses pragmatiques de séquences inscrites dans cette architecture.

L'analyse interlocutoire de séquences (Ghiglione \& Trognon, 1993) repose sur une approche séquentielle et dialogique du discours. Les communications interpersonnelles ont pour caractéristique de prendre forme à travers l'accomplissement de comportements langagiers produits en alternance par les protagonistes. Ce processus d'alternance caractérise les communications interpersonnelles et permet au sens à la fois d'émerger et de se stabiliser. Le processus de fixation du sens se réalise en plusieurs temps et c'est dans l'après-coup conversationnel que le sens se stabilise et que la modalité de construction est alors rendue saillante par l'analyste (Trognon \& Brassac, 1992). L'analyse interlocutoire permet d'étudier la dynamique des interactions langagières traduisant la façon dont le sens des énoncés se construit collectivement au gré des prises de parole. Cette forme d'analyse permet en outre d'accéder à une partie du travail cognitif des interlocuteurs (raisonnements, ressources exploitées) sous-jacent à l'émergence des productions langagières. 


\section{Résultats}

\subsection{La structure globale de la réunion}

L'analyse structuro-fonctionnelle de la réunion permet d'identifier trois phases : une phase préparatoire, une phase évaluative et une phase post-évaluative. La première phase permet de présenter au collectif l'objectif du travail et les consignes pour l'atteindre avec l'explication des quatre critères de cotation et une description du DUER utilisé. La deuxième est la phase principale d'évaluation des risques qui comprend la cotation du risque et de son impact. L'animateur au cours de cette phase guide le collectif en se basant sur le DUER : il soumet les risques génériques et la façon dont ces risques ont été traités l'année précédente. S'ensuit une discussion sur les dangers et le risque évoqué pour enfin mener l'étape de cotation à l'aide des quatre critères. La troisième phase, post-évaluative, permet de rappeler l'importance de la prévention en entreprise et la suite du processus engagé ; elle constitue aussi pour les opérateurs un moment opportun pour ajouter des dangers qui n'auraient pas été évoqués au cours de la deuxième phase.

\subsection{La séquence étudiée}

Dans cette séquence, il est question d'un risque de «chute de plain-pied » ou «accident de plain-pied », en référence à la terminologie couramment employée en France. Les «chutes de plain-pied »sont des accidents du travail souvent perçus comme étant inévitables et de caractère bénin. Contrairement à ces idées reçues, elles sont une des principales causes d'accidents dans l'environnement professionnel. Elles peuvent avoir des conséquences graves, parfois même fatales pour les salariés victimes (Leclercq, 2002 ; Leclercq \& Tissot, 2004). Selon la Commission Européenne, les accidents de plain-pied sont définies comme «les glissades, trébuchements, faux-pas et autres pertes d'équilibre sur une surface "plane" [...] y compris si la victime a pu rétablir son équilibre et qu'il n'y a pas, à proprement parler, chute. Ne sont prises en considération que les surfaces ne présentant aucune rupture de niveau ou bien des ruptures de niveau réduites (trottoir, petites marches, plan incliné, etc.) ». Il s'agit donc d'accident ayant eu lieu à l'occasion d'un travail qui n'a pas été exécuté " en hauteur ». La victime a récupéré son équilibre ou a chuté, en subissant dans les deux cas des lésions. Selon l'approche systémique des accidents, la lésion est l'aboutissement d'une dynamique qui révèle une combinaison de causes en lien avec l'environnement, la tâche, l'organisation du travail, et de causes plus profondes liées au management et au contexte externe à l'entreprise en particulier (Leclercq, 2015). Le concept de perturbation du mouvement (ou de la posture) de la victime intègre la diversité des accidents rencontrés sous l'appellation « accident de plain-pied » (Leclercq, Monteau et Cuny, 2010).

Le risque de chutes dont il est question dans la séquence est lié à l'encombrement du passage, c'est-à-dire lorsque le passage est trop étroit et/ou lorsqu'un obstacle limite la circulation. Le collectif examine la situation des palettes qui sont trop rapprochées de la machine, réduisant l'espace de travail des opérateurs et augmentant la probabilité de se prendre les pieds dans une palette, pouvant provoquer une chute. L'aire de travail des conducteurs est caractérisée par l'écart entre la machine et les palettes, qui contiennent des documents papiers qui seront insérés dans la machine. Parfois, un élément extérieur, appelé le propack, peut être utilisé pour insérer des documents plus épais comme les échantillons de parfum. Lors de son utilisation, le propack est installé dans l'espace de travail contre la machine, réduisant alors le passage. La personne chargée de positionner les palettes est l'approvisionneur. Plus les palettes sont proches de la machine, plus l'espace de travail est réduit et plus le risque de chute liée aux palettes est grand. Ce risque augmente lorsqu'un propack est utilisé puisque celui-ci est installé dans cet espace de travail, qui est parfois réduit lorsque les palettes sont positionnées trop proches de la machine.

\subsection{Le rôle de l'animateur dans cette séquence}

Le rôle de l'animateur ne se réduit pas à guider le collectif sur la base des risques mentionnés dans le DUER. Comme l'illustre la séquence suivante, il cadre l'activité, il explicite, il contextualise, il demande au collectif de s'exprimer sur la base de leur expérience respective.

I 125b : Animateur : y a-t-il possibilité de chute liée à l'encombrement du passage $\uparrow$

I $125 \mathrm{c}$ : circulation entre les palettes et les machines (.) le propack $\downarrow$

I 126 : Opérateur D : ah oui

I 127 : Opérateur $\mathrm{C}:$ ah oui

I 128 : Animateur : donc si vous risquez de tomber (.) parce que devant vous vous avez un obstacle $\uparrow$ bah oui 
I 129 : Opérateur $\mathrm{C}$ : hum

I 130 : Animateur : vous avez les palettes vous avez les machines et vous avez les propacks

I 131 : Opérateur B : le propack on se le prend dans la tête

I 132 : Opérateur D : oui ils mettent des fois les palettes tellement près qu'on ne peut plus passer entre les deux

I 133 : Opérateur B : souvent hein

I 134 : Animateur : ouais puisque ils ne dégagent pas assez derrière $\uparrow$

I 135 : Opérateur B : des fois quand on se baisse

I 136a : Animateur : ouais quand vous vous relevez là (.)

I 136b : mais y'a après vous verrez y’a choc après (2”)

En I 125b, l'animateur initie le traitement d'un risque qui va le conduire en I 128 à soumettre une situation contrefactuelle permettant aux opérateurs de se projeter davantage dans leur cadre professionnel. Certains comportements de l'animateur sont induits par les enchaînements des opérateurs. Par exemple en I 29, le « hum » conduit l'animateur à décrire des éléments de la situation (I 130) pour permettre aux opérateurs de mieux comprendre la notion d'obstacle. L'animateur recadre l'évaluation (I 136b) lorsqu'un opérateur évoque un risque qui n'appartient pas à la catégorie en cours d'appréciation : l'opérateur B évoque la situation où un conducteur s'abaisse près du propack et quand il se relève il peut heurter cet élément, produisant un choc (I 131 et I 135). L'animateur reprécise que cette information ne correspond pas au risque étudié actuellement mais qu'elle sera traitée par la suite.

L'animateur est également en mesure de mener des processus de prévention. La séquence suivante constitue la suite de l'évaluation du risque de chute liée à l'encombrement du passage : le risque est plus important lorsque les palettes, positionnées par l'approvisionneur, sont trop rapprochées de la machine réduisant l'espace de travail de l'opérateur. Les opérateurs ne sont pas responsables de ce risque mais ils subissent les conséquences d'un mauvais positionnement des palettes. Une solution, considérée comme optimale par le responsable émerge néanmoins (I 142) et celle-ci est exprimée à trois reprises lorsque l'animateur affirme que le meilleur moyen est de demander à l'approvisionneur de replacer correctement les palettes (I 143, I 149 et I 151).

I 132 : Opérateur D : oui ils mettent des fois les palettes tellement près qu'on ne peut plus passer entre les deux

I 133 : Opérateur B : souvent hein

I 134 : Animateur : ouais puisque ils ne dégagent pas assez derrière $\uparrow$

(...)

I 136c : donc là ben euh de toute façon qu'est-ce qu'on peut faire $\uparrow$

I 136d : enlever les machines enlever les machines enlever le propack

I 137 : Opérateur $\mathrm{C}$ : ne plus travailler (rire)

I 138 : (rire)

I 139a : Animateur : donc là donc là c'est pareil ben on peut on peut rien faire de mieux à part euh (.)

I 139b: ou alors mettre de la mousse partout

I 140 : Opérateur C : mettre des gyrophares (rire) des klaxons

I 141 : Opérateur B : (rire)

I 142 : Opérateur D : non décaler les palettes tout simplement et pis c'est bon

I 143 : Animateur : oui oui non mais après ça comme je dis toujours il faut il faut lui demander hein

I 144 : Opérateur B : ben des fois les palettes euh elles sont dans un état aussi euh

I 145 : Opérateur $\mathrm{C}$ : hum hum

I 146 : Opérateur B : des sacrés morceaux qui dépassent

I 147 : Animateur : $\uparrow$ ah ben on va le noter (A écrit sur sa feuille)

I 148 : Opérateur B : hier j'en avais une j'étais obligée de donner des coups de pied pour la remettre bien

I 149a : Animateur : comme je l'dis toujours il est préférable d'arrêter la machine cinq minutes (.)

I $149 \mathrm{~b}:$ de demander à l'appro de reculer ses palettes

I 150 : Opérateur D : et pis ceux qu'ont des lunettes le propack il faut faire gaffe hein

I 151a : Animateur : ah ouais non mais voilà il faut dire stop tu dis j'ai pas envie de me mettre en danger donc euh (.)

I 151b : après euh (.) que ça lui plaise ou non à l'appro il euh c'est son métier il doit le faire $\uparrow$

L'enjeu de l'animateur dans cette séquence n'est pas d'instruire un risque mais d'engager les opérateurs dans un changement de pratique dans l'objectif de conduire une action de prévention. La stratégie utilisée par le responsable nous rappelle les travaux de Lewin (1947) et ses enseignements sur l'engagement dans une prise de décision et sur le changement de pratiques. La stratégie est d'amener les opérateurs à évoquer eux-mêmes la 
solution qui marquerait un premier pas vers un engagement à modifier leur pratique. Cette stratégie s'inscrit dans un espace de discussion (Detchessahar, 1997, 2009, 2013) qui constitue un espace de régulation visant la production de solutions. Etudions plus précisément comment opère l'animateur.

L'animateur demande au collectif de trouver une solution (I 136c) pour éviter le danger concernant les palettes mal positionnées. En référence aux pistes données précédemment (I 132 et I 134), le responsable attend une solution faisant référence à l'approvisionneur. Il poursuit son intervention en proposant des solutions non tenables (I 136d et I 139b), ce qui constitue une tentative pour que la solution vienne des opérateurs et non de lui. S'ensuivent d'autres solutions non fonctionnelles à visée humoristique de la part des opérateurs (I 137 et I 140), jusqu'à l'intervention de l'opérateur D (I 142) qui énonce la proposition attendue par l'animateur, en évoquant l'action qui permettra de réduire le danger, c'est-à-dire décaler les palettes en les éloignant. Notons cependant que l'intervention de l'opérateur D n'informe pas sur l'auteur de l'action, ni sur le moyen d'opérationnaliser l'action. Intervient alors l'animateur qui donne cette information manquante (I 143). Soulignons l'emploi du « hein » visant à obtenir un engagement langagier de la part des opérateurs, qu'il n'obtiendra pas.

Les interventions suivantes soulignent l'état de certaines palettes constituant un nouveau danger identifié (I 144 à I 148). Dès ce nouveau danger inscrit sur le document, l'animateur réitère clairement la solution que les opérateurs devraient mettre en œuvre (I 149b) : demander à l'approvisionneur de reculer les palettes. Il renforce ce propos en précisant que la sécurité doit être prioritaire sur la production et que la machine peut être arrêtée si c'est nécessaire (I 149a). L'animateur accentue à nouveau cette solution en indiquant qu'il ne faut pas se mettre en danger (I 151a) et que c'est le rôle de l'approvisionneur de reculer les palettes (I 151b). Manifestement, les opérateurs dans ce type de situation ne font pas ce qui est recommandé pour éviter un risque physique, c'est-à-dire solliciter l'approvisionneur, et par conséquent continuent à prendre des risques physiques.

\subsection{L'arbre des causes}

La figuration suivante permet de restituer les différentes causes pouvant provoquer la situation à risque évoquée dans la partie précédente.

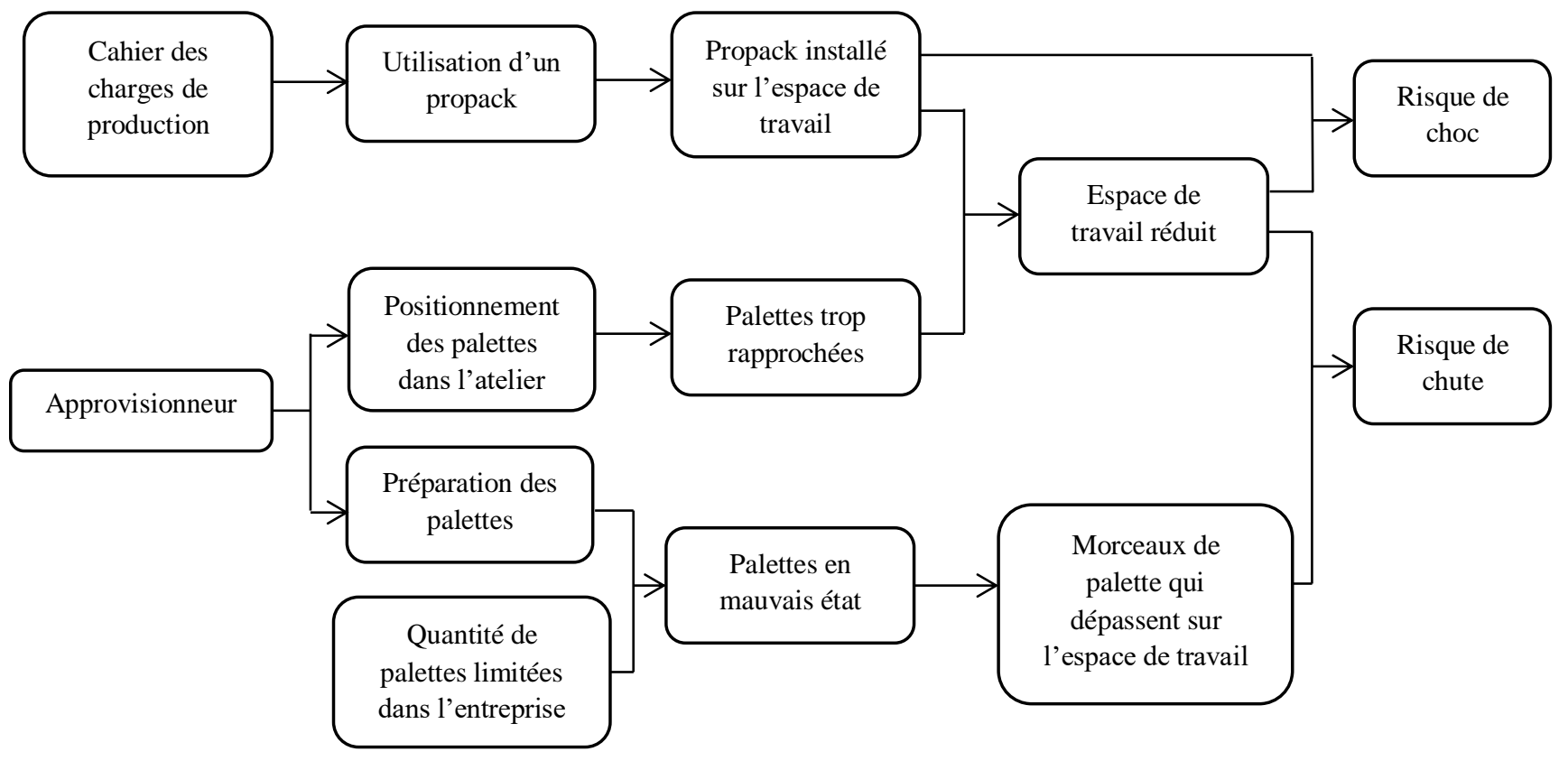

Figure $1:$ L'arbre des causes du risque de chute et de choc

Le risque de chute et de choc est causé notamment par la réduction de l'espace de travail. Cette réduction est liée à la présence du propack et aux palettes trop proches de la machine. Le propack est installé pour répondre à des critères de production en ajoutant dans les plis des échantillons de parfum; il est donc impossible pour les 
opérateurs de réaliser l'activité sans le propack, sinon le cahier des charges ne serait pas respecté. Les palettes sont positionnées par l'approvisionneur ; il est donc l'acteur et l'auteur de cette cause.

Le risque de chute s'explique également par le mauvais état des palettes, laissant des morceaux dépasser. Ces palettes sont préparées par l'approvisionneur qui ne dispose que d'un stock limité. Dans l'immédiat, il n'est donc pas possible de changer ces palettes puisqu'il n'y en pas d'autres.

L'analyse de cette gestion des risques permet d'affirmer que la meilleure solution est de modifier le positionnement des palettes afin d'offrir plus d'espace de travail, limitant alors le risque de chute liée aux palettes et les risques de choc contre le propack. L'approvisionneur apparaît donc comme une source d'augmentation des risques en positionnant mal les palettes et en même temps il constitue la solution en repositionnant correctement celles-ci.

4.5. Le processus communicationnel sous-jacent au traitement du risque

La figure 2 restitue dans sa chronologie les différentes étapes de traitement du risque par le collectif.

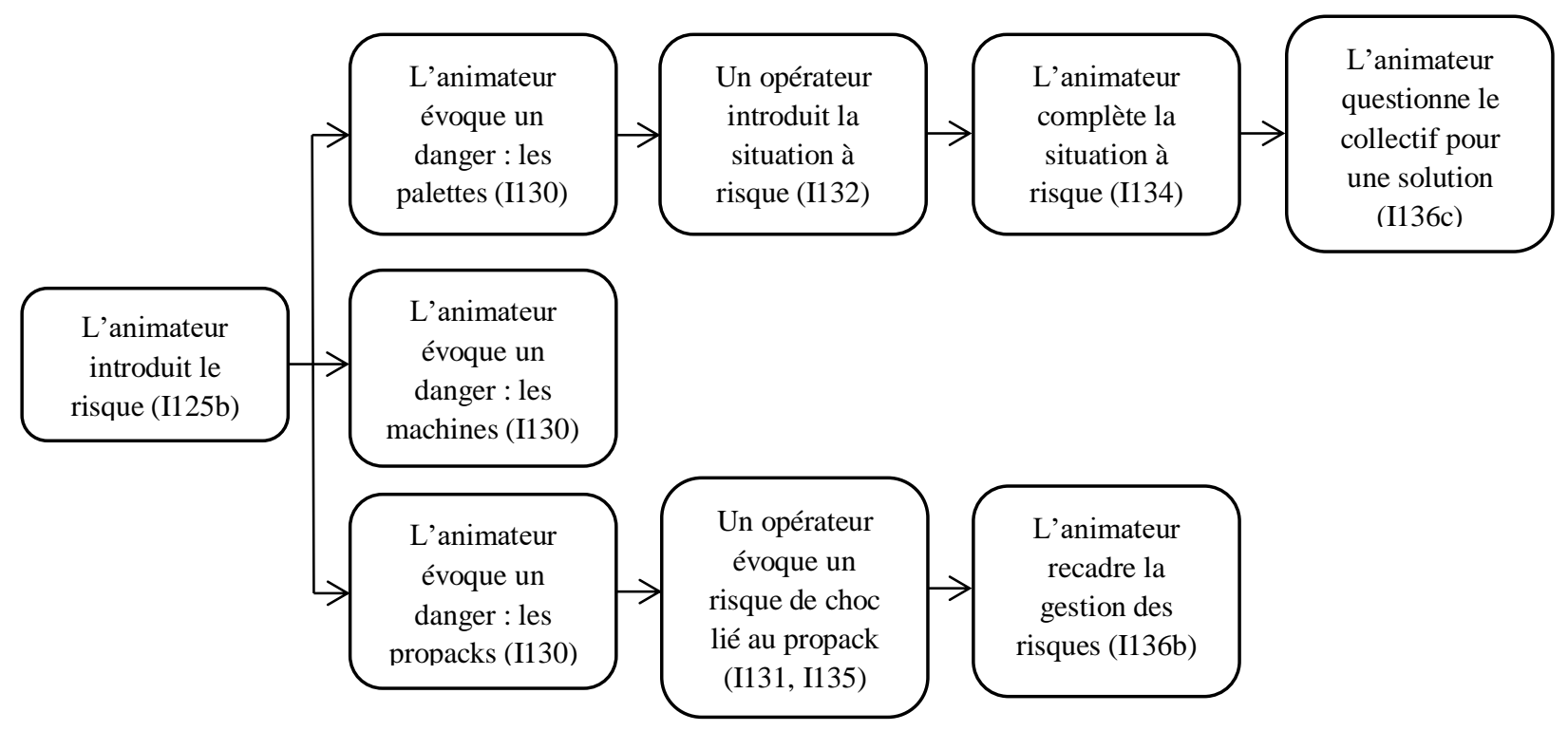

Figure 2 : Le processus sous-jacent à la compréhension du risque

La situation à risque est introduite suite à l'évocation des palettes qui sont considérées comme un danger. L'analyse porte alors sur cette situation orientée vers une solution en lien avec les palettes. 


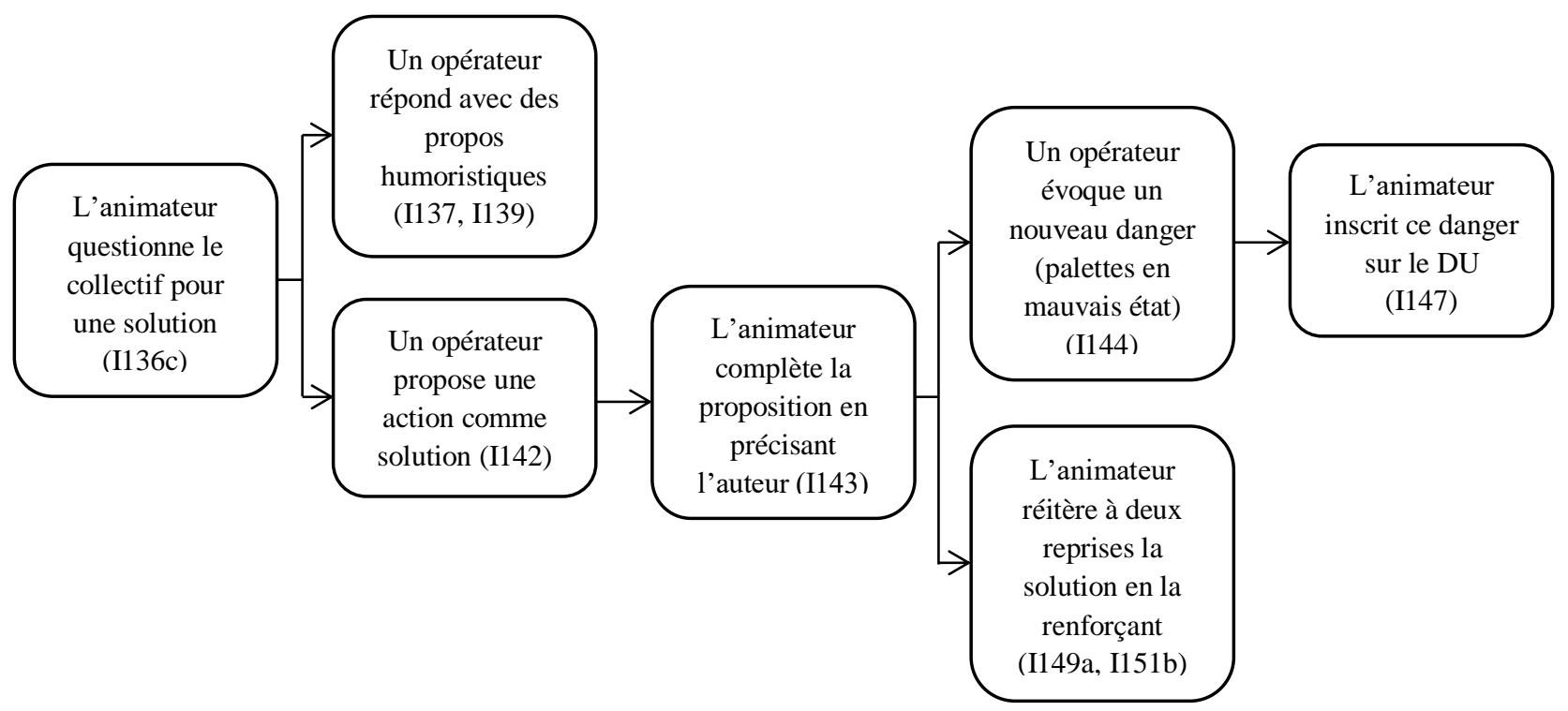

Figure 3 : Le processus de traitement du risque

Le collectif emploie l'humour pour répondre d'une part, puis propose une action afin de répondre à la question posée d'autre part. Mais la proposition de l'opérateur ne précise en aucun cas que c'est l'approvisionneur qui doit reculer les palettes. C'est l'animateur qui complète cette information. En réponse à cette solution, les opérateurs évoquent un nouveau danger, celui des palettes en mauvais état qui sera saisi par l'animateur. Mais ils ne présentent aucun signe d'acceptation de la solution suggérée. Alors l'animateur réitère à deux reprises la demande à faire à l'approvisionneur, en précisant dans un premier temps que la production peut être stoppée pendant ce temps (les opérateurs ont donc la légitimité de stopper la machine pour effectuer la demande et l'action) et dans un second temps que l'approvisionneur n'a pas le choix car le bon positionnement des palettes fait partie de son travail. Mais les opérateurs semblent ne pas vouloir l'appliquer cette solution. Seul l'animateur évoque l'approvisionneur, et les opérateurs ne confirment pas qu'il est l'auteur de la situation problématique et qu'il pourrait être initiateur de la régulation du risque. Au contraire, les opérateurs ciblent l'état des palettes et les chocs liés au propack. La relation entre les opérateurs et l'approvisionneur est peut-être une barrière limitant la prévention du risque ciblé.

\section{Discussion}

La gestion des risques professionnels nécessite de prendre en compte deux difficultés que peuvent rencontrer des opérateurs (Vidal-Gomel, 2007) : l'échec d'identification du danger et des décisions inappropriées de régulation des risques. Dans notre étude, le risque physique est bien identifié mais la solution proposée n'est pas retenue par les opérateurs. Le risque de chute est présent à cause de l'approvisionneur qui positionne les palettes trop proches des machines, réduisant l'espace de travail des conducteurs. L'étude montre que, malgré l'émergence d'une solution (demander à l'approvisionneur de mieux positionner les palettes), la dimension psychosociale empêche l'engagement des salariés dans l'application de la solution. On observe un phénomène décrit en psychologie sociale, l'effet Janis (1972) qui consiste à privilégier une décision dont on sait qu'elle est mauvaise ou non optimum au bénéfice du maintien d'un climat social serein. En effet, lorsque le collectif échange sur les risques de chutes liées à l'encombrement du passage, une situation de danger est évoquée : si des palettes sont positionnées trop proches de la machine, alors l'espace de travail est réduit et le risque de chute est augmenté. L'approvisionneur étant chargé de placer les palettes, une solution émerge et vise à demander à l'approvisionneur de reculer les palettes ; néanmoins les opérateurs n'adhèrent pas à cette option.

Un entretien a posteriori a été conduit avec l'animateur et a permis de déceler que les opérateurs craignaient l'approvisionneur. En effet, il semble fréquent que l'approvisionneur exprime un tempérament fort, 
voire de la mauvaise humeur, provoquant ainsi une appréhension des conducteurs à faire appel à lui. Ainsi, les opérateurs préfèrent tolérer un risque physique lié aux palettes trop rapprochées plutôt qu'un risque « d'agression » de la part de l'approvisionneur. Ainsi, l'étude montre que, même si l'animateur suggère une solution applicable requérant un éventuel arrêt de la production qu'il autorise en sa qualité de responsable sécurité et production de l'entreprise, son statut hiérarchique n'est pas suffisant pour engager des opérateurs dans la mise en place de la solution trouvée. Ils préfèrent prendre un risque physique plutôt que de s'exposer à un risque psychosocial (Amalberti, 1996). Ce résultat est à mettre en lien avec les travaux de Grosjean et Ribert-Van de Weerdt (2010) qui montrent que les émotions pressenties peuvent impacter la situation de travail et orienter des prises de décision. Les prises de décision reposent sur les ressources mobilisées par les opérateurs au sens des modèles transactionnels de Lazarus et Folkman (1984) afin d'identifier les avantages et les inconvénients de la situation dommageable pour prendre une décision pouvant d'ailleurs impliquer un « risque acceptable » (Fischhoff, Lichtenstein, Slovic, Derby \& Keeney, 1981 ; Cadet \& Kouabénan, 2005).

\section{Conclusion}

L'étude de cas débouche sur différents enseignements.

Si le processus d'identification des risques (fondés sur l'emploi d'outils comme les questionnaires) induit une dichotomie entre risques physiques et risques psychosociaux, cette distinction doit disparaître lorsqu'il s'agit de développer des actions visant la réduction ou la suppression des risques. La prévention des risques gagne à être intégrée dans une gestion globale, un management intégré qui prend en compte les interactions possibles entre les différents risques. Aussi les animateurs de tels groupes doivent être formés à l'identification de l'expression de risques psychosociaux même si le guide (par exemple le DUER existant) induit un travail sur les risques physiques. Les animateurs doivent donc être attentifs à l'expression de ces risques psychosociaux et à leur formalisation car ils devront intégrer le DUER. Ils doivent aussi être formés à la production de relances visant à instruire davantage le versant socio-émotionnel des situations investies qu'elles soient factuelles ou contrefactuelles.

On identifie également l'importance de créer des groupes pour le traitement des risques professionnels. Ces groupes permettent d'investir collectivement chacune des situations à risques, d'identifier leurs causes et de pouvoir partager une compréhension des situations, nécessaires à des solutions tenables ; mais aussi d'envisager et réfléchir aux situations générées par la mise en place de solutions - ces réflexions collectives pouvant déboucher sur l'identification et/ou l'émergence de risques non envisagés antérieurement.

En outre la prise de décision concernant la mise en place d'une solution peut être influencée par différents critères, comme l'expérience, les états mentaux et émotionnels, les connaissances. La prise de décision va dépendre aussi de ce qui se négocie, se coconstruit au cours de la discussion, sachant que les opérateurs peuvent ne pas percevoir un risque et son acceptabilité de la même manière (Kouabénan, 2007, 2009 ; Kmiec \& Roland-Levy, 2014). Certains peuvent considérer un risque comme tolérable, gérable ou bénéfique, alors que d'autres peuvent le percevoir comme intolérable, ingérable ou dommageable. Ces réunions permettent alors de stabiliser la représentation de la situation qui se trouve argumentée et justifiée et de déboucher davantage sur des solutions consensuelles.

Enfin l'absence de l'approvisionneur dans le groupe - puisque n'appartenant pas à l'unité - qui n'a pas permis l'adhésion à une solution au risque physique, invite à s'interroger sur la composition des groupes d'opérateurs sollicités pour l'identification et la régulation des risques. Il conviendrait en amont d'étudier l'organisation du travail, identifier les relations inter et intra-équipe pour solliciter les représentants les plus pertinents relativement aux risques professionnels à instruire. Cette étude préalable pourrait par exemple s'inspirer de la méthodologie développée par Ashoori, Burns, d'Entremont et Momtahan (2014) (team Cognitive WorkAnalysis) pour l'étude des activités de travail dans les organisations.

Les auteurs déclarent ne pas avoir de lien d'intérêts. 


\section{Bibliographie}

Althaus, V., Grosjean, V., \& Brangier, E. (2012). L'intervention systémique en santé et bien-être au travail : cadre et forme d'une intervention sur la gestion des âges. Activités, 10 (1), 127-149.

Amalberti, R. (1996). La Conduite de systèmes à risques. Paris : Presses Universitaires de France.

Andéol, B., Guillemy, N., \& Leroy, A. (2010). Evaluation des risques professionnels. Questions-réponses sur le document-unique. ED 887, 20 p.

Anzieu, D., \& Martin, J.-Y. (1968). La dynamique des groupes restreints. Paris : Presses Universitaires de France.

Aublet-Cuvelier, A. (2011). Quelles relations entre TMS et RPS ? 3ème congrès francophone sur les troubles musculosquelettiques, Grenoble, 26-27 mai.

Ashoori, M., Burns, C.M., d'Entremont, B., \& Momtahan, K. (2014). Using team cognitive work analysis to reveal healthcare team interactions in a birthing unit. Ergonomics, 57(7), 973-986.

Buono, A. F, \& Jamieson, D. W. (2010). Consultation for Organizational Change. Charlotte, NC: Information Age Publishing.

Cadet, B. \& Kouabenan, D. R. (2005). Évaluer et modéliser les risques : apports et limites de différents paradigmes dans le diagnostic de sécurité. Le travail humain, 68(1), 7-35.

COCT (Conseil d'orientation sur les conditions de travail). (2013). Pathologies d'origine psychique d'origine professionnelle. Partie 1 : pathologies psychiques. Rapport final. Références en santé au travail,133, pp. 75-86.

Dares (2014). Les facteurs psychosociaux en France et en Europe. Darès Analyses, publication de la direction de l'animation de la recherche, des études et des statistiques, $n^{\circ} 100,7 \mathrm{p}$.

Detchessahar, M. (2013). Faire face aux risques psycho-sociaux : quelques éléments d'un management par la discussion. Négociations, 19(1), 57-80.

Detchessahar, M. (2009). Les déterminants organisationnels et managériaux de la santé au travail : l'enjeu de la parole sur le travail. Rapport pour l'Agence Nationale de la Recherche, 766 pages.

Detchessahar, M. (1999). Eléments pour une théorie de l'espace de discussion en situation de gestion. Thèse de doctorat en Sciences de gestion de l'Université de Rennes 1.

Dumez, H. (2011). Qu'est-ce que la recherche qualitative ? Le Libellio d'Aegis, 7(4), 47-58.

Fischhoff, B., Lichtenstein, S., Slovic, P. Derby, S. L., \& Keeney, R. L. (1981). Acceptable Risk. Cambridge: Cambridge University Press.

Ghiglione, R., \& Trognon, A. (1993). Où va la pragmatique? Grenoble : Presses universitaires de Grenoble.

Grosjean, V., \& Ribert-Van de Weerdt, C. (2010). Identité et travail émotionnel dans la relation de service : le cas particulier des centres d'appels téléphoniques. In M. Dubois, D. Retour, \& M.-E. Bobillier Chaumon (Eds), La Relation de service : nouveaux usages, nouveaux acteurs. Bruxelles : De Boeck.

Janis, I. (1972), Victims of Groupthink: A Psychological Study of Foreign-Policy Decisions and Fiascoes, Boston: Houghton Mifflin.

Jeoffrion, C., Hamard, J.-P., Barre, S., \& Boudoukha, A.-H. (2014). Diagnostic organisationnel et prévention des risques psychosociaux dans un établissement d'accueil pour personnes âgées : l'intérêt d'une méthodologie mixte et participative. Le travail humain, 4(77), 373-399. 
Karasek, R. A., \& Theorell, T. (1990). Healthy work. Stress, productivity, and the reconstruction of working life. New York: Basic Book.

Kmiec, R., \& Roland-Lévy, C. (2014). Risque et construction sociale : une approche interculturelle. Les cahiers internationaux de psychologie sociale, 101(1), 69-99.

Kop, J.L, Grosjean, V., Formet, N., \& Althaus, V. (2013). Approche «bien-être au travail » pour la prévention des RPS. Satin (version2.1) - Questionnaire d'évaluation des conditions de travail et de la santé. Démarche de validation. Notes scientifiques et technique de l'INRS, 300. Repéré sur : http://www.inrs.fr/inrs/recherche/etudespublications-communications/doc/publication.html?refINRS=NOETUDE/P2012-212/NS\%20300

Kostulski, K. \& Trognon, A. (1998). Le domaine cognitif de l'interlocution : un exercice d'analyse interlocutoire d'une transmission orale dans une équipe paramédicale. In K. Kostulski, \& A. Trognon (Eds.), Communications interactives dans les groupes de travail (pp. 59-101). Nancy : Presses Universitaires de Nancy.

Kouabénan, D.R. (2009). Role of beliefs in accident and risk analysis and prevention. Safety Science, 47(6), 767776.

Kouabénan, D.R. (2007). Incertitude, croyances et management de la sécurité. Le travail humain, 70(3), 271-287.

Lacoste, M. (1983). Des situations de parole aux activités interprétatives. Psychologie Française, 28(3/4), 231238.

Leclercq, S. (2015). Chutes, heurts et autre perturbations du mouvement, les comprendre pour les prévenir. Enjeux pour la sécurité au travail et freins à la prévention. Hygiène et Sécurité du Travail, 239, 24-27.

Leclercq, S. (2002). Prevention of Falls on the Level in Occupational Situations: A Major Issue, a Risk to be Managed. International Journal of Occupational Safety and Ergonomics, 8(3), 377-385.

Leclercq, S., Monteau, M., \& Cuny, X. (2010). Avancée dans la prévention des « chutes de plain-pied » au travail. Proposition de définition opérationnelle d'une nouvelle classe : « les Accidents avec Perturbation du Mouvement (APM) ». Perspectives interdisciplinaires sur le travail et la santé, 12 (3), http://pistes.revues.org/2667 ; DOI : 10.4000/pistes.2667

Leclercq, S., \& Tissot, C. (2004). Les chutes de plain-pied en situation professionnelle. Circonstances de chutes particulièrement graves à travers l'analyse statistique de 459 cas. Hygiène et Sécurité du Travail, Cahiers de Notes Documentaires, 194 (4), 51-66.

Lewin, K. (1947). Group decision and social change. In T. Newcomb, \& E. Hartley (Eds.), Readings in Social Psychology (pp. 340-344). New York: Holt.

Malenfer, M. (2014). Evaluer les risques professionnels dans les TPE : les atouts du numérique. Hygiène et Sécurite du Travail, 234, 27-32.

Monteau, M. (1974). Méthode pratique de recherche de facteurs d'accidents. Principes et application expérimentale, rapport INRS R140/RE.

Mucchielli, R. (1992). La dynamique des groupes (13 éd.). Paris : ESF éditeur.

Roulet, E., Auchlin, A., Moeschler, J., Rubbattel, C., \& Schelling, M. (1985). L'articulation du discours en français contemporain. Berne : Peter Lang.

Rossignol, K., Drais, E., \& Farvaro, M. (2016). Evaluer l'organisation de la santé-sécurité au travail. Trajectoire d'un outil de mesure au sein du système français de prévention. Terrains \& travaux, 28, 153-173.

Sarnin, P., Caroly, S., \& Douillet, P., (2011). Contre les « risques » psychosociaux, quel débat sur l'activité ? Le travail humain, 74(4), 309-320. 
Stock, S., Nicolakakis, N., Messing, K., Turcot, A., \& Raiq, H. (2013). Quelle est la relation entre les troubles musculo-squelettiques (TMS) liés au travail et les facteurs psychosociaux ? Perspectives interdisciplinaires sur le travail et la santé [En ligne], 15-2 | 2013, mis en ligne le 01 août 2013, URL : http://pistes.revues.org/3407 ; DOI : 10.4000/pistes.3407

Toulouse, G., St-Arnaud, L., Duhalde, D., Lévesque, J., Delisle, A., \& Comtois, A.S. (2013). Diagnostic ergonomique résultant de la présence de risques psychosociaux contribuant aux troubles musculo-squelettiques : le cas des centres d'appels d'urgence 9-1-1. Perspectives interdisciplinaires sur le travail et la santé [En ligne], 15-2 | 2013, mis en ligne le 01 août 2013, URL : http://pistes.revues.org/3385 ; DOI : 10.4000/pistes.3385

Trognon, A., \& Brassac, C. (1992). L’enchaînement conversationnel. Cahiers de Linguistique Française, 13, 76-107.

Vidal-Gomel, C. (2007). Compétences pour gérer les risques professionnels : un exemple dans le domaine de la maintenance des systèmes électriques. Le travail humain, 70(2), 153-194. 